\title{
Acceso a la justicia internacional: una lectura transversal del CIADI y el OSD de la OMC
}

\section{International Access to Justice: A Cross-Reading of ICSID and WTO DSB}

Fecha de recepción: 20 de agosto de 2010 Fecha de aprobación: 18 de noviembre de 2010

\begin{abstract}
Germán Burgos**
\section{Resumen}

Este artículo identifica un conjunto de tendencias transversales que caracterizan el acceso a la justicia de los Estados del tercer mundo en el CIADI del Banco Mundial y el OSD de la OMC. En tal sentido, plantea un conjunto de hipótesis sobre lo que serían las principales oportunidades y obstáculos para dicho acceso a partir de un marco analítico que ubica las particularidades que tiene la justicia internacional en comparación con la administración de justicia de tipo nacional.
\end{abstract}

Palabras clave: acceso a la justicia, justicia internacional, CIADI, Banco Mundial, OMC.

El presente artículo es producto del proyecto de investigación presentado a la Universidad Católica de Colombia intitulado “Estado, Globalización y Administración de Justicia".

** Profesor de la Universidad Nacional de Colombia y de la Universidad Católica de Colombia. 


\begin{abstract}
This article identifies some trends in the access to justice of third world states in the ICSID of the World Bank and the WTO dispute settlement mechanism. In that sense it approaches a group of hypothesis about opportunities and obstacles for this access taking into account an analytical framework that recognizes the particularities of international justices in comparison to national ones.
\end{abstract}

Key words: access to justice, international justices, ICSDI, World Bank, WTO.

\section{INTRODUCCIÓN}

El acceso a la justicia es uno de los temas que ha centrado el proceso de reformas y fortalecimiento de la administración de justicia en América Latina en las últimas tres décadas. En tal sentido, se han introducido, entre otros, un conjunto importante de cambios institucionales tales como un sistema de defensoría pública más profesional e independiente; se han admitido legalmente diversos mecanismos alternativos de solución de controversias; se han reformado los procedimientos para hacerlos más ágiles y accesibles. Se han implementado variados tipos de proyectos piloto para acercar el servicio de justicia mediante mecanismos como las casas de justicia o las ventanillas multipropósito (Dakolias 1995; Bou, 2001 \& Domingo, 2001).

La preocupación por el acceso a la justicia se ha ubicado en el marco general del fortalecimiento de la defensa de los derechos humanos. En este sentido, se ha partido de considerar que garantizar un mejor y mayor acceso a la justicia es un medio necesario para obtener la garantía última de los derechos (Smith, 2007). A pesar de la relativa novedad de la anterior discusión en América Latina, la preocupación por el tema de los obstáculos al acceso a la justicia surgiría en el contexto de las reformas a la justicia en el marco de los estados de bienestar europeo y estadounidense. En particular bajo los trabajos de Capelleti y Garth se logró identificar cuáles eran las principales limitaciones que tenían los ciudadanos para acceder al sistema de justicia y por esta vía hacer valer sus derechos particularmente económicos y sociales ante la administración (Capelleti \& Garth, 1996). Debe anotarse que bajo el momento liberal del Estado moderno, el tema del acceso a la justicia se asumía como un asunto estrictamente individual.

Los desarrollos en torno al acceso a la justicia en la actualidad, no sólo han fortalecido su dimensión institucional sino su carácter en cuanto derecho. En efecto, la Convención Interamericana de Derechos Humanos reconoce, entre otros, que "toda persona tiene derecho a un recurso sencillo y rápido o a cualquier otro recurso efectivo ante los jueces o tribunales competentes, que la ampare contra actos que violen sus derechos fundamentales" (art. 25). Por su parte, el artículo 8 del mismo instrumento, indica que "toda persona tiene derecho a ser oída, con las debidas garantías y dentro de un plazo razonable, por un juez o tribunal competente, independiente e imparcial" (Acosta, 2007).

Los avances jurídicos en torno de la dimensión como derecho del acceso a la justicia, también tienen expresión a nivel jurídico interno sea constitucional como legalmente. Por ejemplo, en Colombia la Carta Superior reconoce en su artículo 229, que se garantiza el derecho de toda persona para acceder a la administración de justicia. Por su parte, la ley estatutaria de la administración de justicia, indica como

El Estado garantiza el acceso de todos los asociados a la administración de justicia. Será de su cargo el amparo de pobreza y el servicio de defensoría pública. En cada municipio habrá como mínimo un defensor público (art. 2). 
El gran vacío en el desarrollo de esta temática remite al casi nulo análisis sobre las condiciones de acceso a la justicia internacional. En años recientes, los espacios de este tipo de justicia por fuera del ámbito del Estado-nación, han crecido de manera exponencial. Hoy, en efecto, contamos no sólo con la ya tradicional Corte Internacional de Justicia sino con diversos espacios de solución de controversias en los ámbitos de los derechos humanos, el comercio internacional, las inversiones, etc. En algunos de estos ámbitos ya no sólo son los Estados los que pueden llevar causas a su conocimiento, también los individuos cuentan con vías para acceder a la justicia internacional.

A pesar de esto, el estudio sobre las posibilidades y obstáculos para acceder a la justicia internacional no ha concitado el interés de los internacionalistas o de los especialistas en el tema del acceso a la justicia. Solo en los casos del sistema interamericano y del órgano de solución de controversias de la OMC se pudo verificar algún tipo de investigaciones sobre las condiciones de acceso de las víctimas y los estados respectivamente.

Las razones para el anterior vacío son varias. De un lado, la justicia internacional se concibe en general como complementaria o subsidiaria de la justicia nacional, por tanto, tiene un carácter excepcional. En segundo lugar y con matices para el caso de los derechos humanos, son los Estados los que ante todo pueden usar los mecanismos de solución de controversias, lo cual al parecer hace presumir que aquellos no tienen mayor dificultad para acceder. Finalmente, siempre está la inquietud sobre el nivel de eficacia de estos mecanismos, la cual al ser relativamente baja, puede conllevar un limitado interés en razón de su impacto.

El presente artículo pretende llenar parcialmente el anterior vacío. En tal sentido, su objetivo inicial es plantear un marco analítico sobre cómo analizar el estado del acceso a la justicia internacional a partir de la identificación de un conjunto de variables tipo identificadas para el efecto. Adicionalmente y como segunda pretensión, se presentarán un conjunto de hipótesis transversales sobre lo que pueden considerarse las principales oportunidades y obstáculos al acceso a la solución de conflictos en el CIADI del Banco Mundial y en el órgano de solución de diferencias de la OMC vistos a la luz de la situación general de los países del tercer mundo ${ }^{3}$.

El presente artículo está organizado en tres partes. La primera de ellas da cuenta del marco analítico sobre el acceso a la justicia, haciendo una especial referencia a algunas particularidades de la justicia internacional. A continuación se identificarán las tesis centrales sobre los organismos objeto de estudio, presentadas de manera transversal. Finalmente se ofrece un conjunto de conclusiones.

\section{EL ACCESO A LA JUSTICIA INTERNACIONAL: UNA CONCEPTUALIZACIÓN}

El contenido de lo que entender por acceso a la justicia en sentido integral, es decir, más allá de su carácter de derecho, se ha venido ampliando de manera paulatina. Inicialmente se entendió que aquel era un problema relacionado con las barreras y oportunidades para usar el sistema formal de cortes. En otros términos, acceder a la justicia significaba tener las condiciones legales, económicas y físicas para dar a conocer al aparato de justicia determinado conflicto o reivindicación de tipo jurídico.

Posteriormente, el concepto de acceso se fue complejizando en la medida que se empezó a plantear que no bastaba con presentar un caso al sistema judicial. Se precisaba, de la misma manera, contar con un trámite efectivo y garantista que permitiera contar con una decisión de fondo

3 Parte de la información aquí recogida es producto de un proyecto de investigación financiado por la Agencia de Cooperación Alemana (GTZ) durante 2009. 
en torno a lo solicitado. Por esta vía el acceso a la justicia se cualificó en la medida que no era un problema sólo de oportunidad de presentar sino de contar con una decisión a tiempo y respetuosa del debido proceso (UNDP, 2004).

Finalmente, el anterior cuadro se hizo aún más integral al tenerse en cuenta que se empezó igualmente a defender que acceder a la justicia conllevaba contar con una decisión efectiva. Es decir, se precisa como parte del acceso, que se debe garantizar la ejecución debida de lo decidido judicial o extrajudicialmente (Moncayo, 1995 \& Moreno, 2000).

Adicionalmente, la discusión sobre el acceso a la justicia introdujo progresivamente el tema de los mecanismos alternativos de solución de controversias. En concreto se ha venido planteando, $y$ hoy es generalmente aceptado, que la resolución de fondo y efectiva de los conflictos o el reconocimiento de derechos, puede ocurrir mediante mecanismos de justicia formal (cortes en sus diversos niveles) o informal (mecanismos de conciliación, mediación, etc.) (Vigoriti, 2008).

Es claro que lo visto se ha construido a partir de considerar el acceso a la justicia a nivel nacional antes que internacional. En otros términos, las especificidades de la justicia global o internacional no han sido tenidas en cuenta a la hora de construir el concepto de acceso a la justicia. Estas se han basado en los referentes del Estado-nación y su administración de justicia.

A partir de lo anterior, para nuestros efectos, el acceso a la justicia se entenderá en los siguientes términos: posibilidad efectiva de usar los mecanismos formales e informales para solucionar a tiempo y con la garantía del debido proceso, conflictos $y / 0$ garantizar derechos individuales y/o colectivos a nivel internacional y/o global. Al tener en cuenta esta definición que incluye el antes, el durante y el después del trámite de una causa judicial o extrajudicial, el presente marco analítico ha identificado un conjunto de variables que constituyen simultáneamente oportunidades y/o obstáculos para el acceso a la justicia. En otros términos, vistos en positivo son posibilidades para acceder a los mecanismos de solución de controversias y valorados en negativo son límites (CIDH, 2007 \& DPLF, 2008).

Centrándonos en las variables, estas se visualizan según lo que denominamos fases del acceso a la justicia internacional. En estos términos, la fase uno, correspondiente a las posibilidades de usar o no un determinado mecanismo de solución de controversias, también denominada fase anterior al ingreso, nos permitió determinar las siguientes variables que pueden influir en el acceso:

- Reconocimiento legal de un derecho o deber y de vías para tramitarlo formal o informalmente.

- Justicia no costosa.

- Acceso a asistencia legal.

- Confianza en los sistemas de justicia.

- Idioma del sistema accesible.

- Bajo nivel de formalismo.

- Cercanía física.

- No discriminación.

En consecuencia, serían obstáculos para llegar a los mecanismos de solución:

- Ausencia de conciencia jurídica.

- No existencia de vías institucionales para defender un derecho o reivindicación jurídica.

- No asistencia legal o de baja calidad.

- Costo alto de los mecanismos.

- Lejanía de las oficinas judiciales.

- Desconfianza.

- Formalismo jurídico.

- Idioma inaccesible o difícil.

- Discriminación. 
Por su parte, la fase dos, relacionada con el acceso, corresponde al trámite del conflicto luego de haber sido admitido por la correspondiente instancia de solución de controversias. En tal sentido, las variables tenidas en cuenta como oportunidades y obstáculos son:

- Eficiencia y eficacia.

- No formalismo.

- Debido proceso.

- Asistencia legal.

- Imparcialidad.

- Pulcritud.

- Pluralidad.

- Acceso a recursos respecto de la decisión.

Vista en clave de obstáculos estos serían:

- Ineficiencia e ineficacia.

- Formalismo.

- No garantías.

- Inasistencia legal.

- Corrupción.

- Discriminación o parcialidad.

- No acceso a recursos en contra de las decisiones.

En último lugar, como se definió antes, el acceso a la justicia se garantiza en la medida en que la decisión pueda ser viable en su cumplimiento y respeto por los actores. Esta corresponde a la fase 3 , también conocida como posterior al trámite y las variables a analizar son:

- Resolución a tiempo.

- Decisión que respete derechos.

- Decisión que resuelva el fondo.

- Cumplimiento de la decisión.

Serían en tal sentido los siguientes obstáculos:

- Resolución tardía.

- Decisión no respetuosa de los derechos.
- Resolución no de fondo.

- Decisión no cumplida total o parcialmente.

A partir de las variables antes identificadas, el presente artículo pretende identificar hasta qué punto las oportunidades de acceso se cumplen o por el contrario lo que existe son obstáculos de diverso tipo al acceso a la justicia internacional o global. De manera específica, se busca determinar las posibilidades y dificultades de acceso de los Estados en materia de las instancias del derecho económico global arriba identificadas.

A este respecto, debe anotarse que dado el carácter amplio y complejo del concepto de acceso tomado aquí y la amplitud de variables consideradas, no siempre es fácil encontrar información suficiente que permita ubicar el comportamiento de todos los factores que queríamos tener en cuenta. Esto último, en parte, se explica porque la investigación se fundó en fuentes secundarias, las cuales no siempre abordaban las variables antes descritas. Por tanto, se logró en varios casos identificar hipótesis en materia de oportunidades y obstáculos, pero en algunos casos no hubo información suficiente para establecer mínimamente alguna tendencia. Aún en aquellas situaciones donde se logró identificar esta última, el tipo de información identificada apenas nos permitió construir algunas hipótesis que merecen un nivel de confirmación o cuestionamiento más profundo en el futuro.

\section{PARTICULARIDADES DE LA JUSTICIA INTERNACIONAL Y/O GLOBAL}

Teniendo en cuenta lo antes descrito, la investigación asociada a este artículo parte de considerar que la justicia internacional o global tiene algunas particularidades que la diferencian de su dimensión nacional por lo menos a la hora de analizar el acceso. Estas deben ser valoradas al momento del análisis. Dichas particularidades se pueden identificar en los siguientes términos: 
1. La justicia internacional o global tiende a ser excepcional al menos para los individuos, aunque esto es cada vez menos claro. En otros términos, al menos para los casos objeto de análisis, una parte de los mecanismos analizados precisan de alguna manera el agotamiento de las vías internas. Sin embargo, si tenemos en cuenta los casos del OSD de la OMC y del arbitraje comercial del Estado, existe acceso directo.

2. A pesar de la mayor o menor "inmediatez "del acceso, este a nivel internacional es por definición restringido. En otros términos, a diferencia de la justicia nacional donde todos los ciudadanos pueden en abstracto usar el sistema de justicia, en términos internacionales o globales los sujetos calificados son restringidos. Estos fundamentalmente corresponde ante todo a los Estados-nación y de manera creciente aunque excepcional a los individuos. En suma, la accesibilidad en la arena internacional es por definición limitada.

3. Como se ha indicado en el punto anterior, son los Estados los que de manera dominante tienen mecanismos internacionales para acceder a la justicia. Sin embargo, en muchos casos su libertad para optar o no por estos mecanismos está limitada. Así por ejemplo, el uso de los mecanismos del órgano de solución de controversias de la OMC es de carácter obligatorio para cualquier miembro de este organismo y para el caso del CIADI su uso es cada vez mayor como producto de la negociación de los tratados bilaterales de inversión. Si bien Ios Estados tienen la posibilidad de construir $y$, hasta cierto punto, escoger los escenarios donde resuelven los conflictos internacionales en que se ven inmersos según la materia, este proceso está sujeto a distintos niveles de constricciones sean producto de los mismos tratados o de negociaciones no siempre desarrolladas en condiciones igualitarias.
4. A diferencia del derecho interno o nacional, el derecho internacional público no reconoce un derecho de acceso a la justicia, a los sujetos internacionales. En efecto, dado el carácter excepcional de estos mecanismos, el acceso dominante de los Estados y el excepcional de los individuos, hoy no contamos con un derecho jurídicamente reconocido para poder acceder de manera universal a espacios de solución de controversias. Lo anterior es hasta cierto punto entendible dado el carácter cerrado de los sistemas de solución y el hecho de que en algunos casos los estados se muestran renuentes a acceder a determinadas instancias en las cuales no confían.

5. Como se registrará los espacios de solución de conflictos internacionales en materia de derecho económico internacional, han ganado un importante poder e importancia especialmente respecto de los Estados. Cada vez más existen mecanismos para obligar a estos a usar y reconocer las decisiones de instancias internacionales. Claramente, los casos del OSD de la OMC, serían los más emblemáticos. A pesar de lo anterior, esta investigación parte y finalmente confirma que la efectividad de las decisiones tomadas por instancias internacionales, descansa en gran parte en la acción del Estado. Por tanto, como se demuestra en la investigación, estos sujetos internacionales tienen formas de resistirse o administrar el cumplimiento parcial o limitado de las decisiones internacionales y/o globales.

\section{EL ACCESO A LA JUSTICIA INTERNACIONAL. TENDENCIAS E HIPÓTESIS}

Según se registró con antelación, a continuación se presentan de manera resumida un conjunto de hipótesis sobre oportunidades y obstáculos para el acceso al CIADI del Banco Mundial y al OSD de la OMC. 


\section{Acceso a la justicia antes de la presentación de un caso}

Como se acotó en el punto anterior, el acceso antes de trabar jurídicamente un conflicto, está enmarcado en a qué sujetos se le reconoce legitimidad para acceder a la justicia global. En líneas generales son los Estados, seguidos por los sujetos individuales, titulares de derechos individuales y/o económicos. Obviamente esto depende del ámbito del que hablemos. Contrario sensu, la sociedad civil vía otros actores no tiene reconocido en principio el acceso, salvo con las figuras del Amicus curie admitidas de manera excepcional para el CIADI y la OMC (Cruz, 2002; Odumosu, s.f. \& Peterson, 2003).

En líneas generales, la posibilidad de presentar una pretensión o demanda en las instancias analizadas está facilitada por lo que es un escaso nivel de formalización para usar el sistema. Esto quiere decir que el procedimiento no tiene rigideces excesivas para poner en consideración unos determinados hechos. A esto se le suma que en el CIADI, se puede poner en consideración un caso usando las Nuevas Tecnologías de la Información y las Telecomunicaciones -NTIC. Adicionalmente, como oportunidades en este terreno está la flexibilidad en la adopción de los procedimientos. Como las partes pueden escoger el procedimiento a seguir esto puede facilitar igualmente su ajuste a la hora de su desarrollo (Hernández, 2009).

Los obstáculos para el acceso, visto desde los Estados son varios. En primer lugar, en algunos casos estos no pueden llevar la iniciativa en la presentación de una pretensión, como ocurre por ejemplo en caso del CIADI, que según la forma como se prevea su competencia, solo permite la acción a los inversionistas extranjeros. En otros casos, como los de la OMC, existe un obstáculo fáctico particularmente para los países del mundo en desarrollo. Si bien muchos de ellos pueden considerar violatorias de los acuerdos de las OMC determinadas decisiones de otros Estados, especialmente desarrollados, no siempre presentan una reclamación por temor a represalias o reacciones contrarias que pongan en tela de juicio las condiciones de su comercio internacional. Existe, en los dos casos enunciados, una desigualdad de origen para el acceso, la una jurídica y la otra fáctica. Finalmente y volviendo a la $\mathrm{OMC}$, la posibilidad de participación en un caso como tercer interviniente, está supeditada no a los efectos de un determinado hecho contario a los tratados comerciales, sino al volumen de comercio que un Estado tenga respecto de determinado sector. (Arenas, 2009).

Ya en términos económicos, el acceso está obstaculizado por distintas vías. Algunos mecanismos para ser usados precisan del pago de un valor determinado. Es lo que ocurre con el CIADI y los distintos mecanismos de arbitraje comercial internacional, aunque debe registrarse que para el primer caso, quien cubre estos costos es generalmente la empresa que inicia la acción (Hernández, 2009). En segundo plano están los costos económicos asociados a la contratación de los abogados para presentar un caso o responder una demanda. Si bien la información sobre el costo por hora de estos servicios no es siempre pública, los costos son por lo general altos. A este nivel debe mencionarse el particular caso de la OMC, el cual incluye la existencia de un centro de asistencia legal, especialmente, dirigido a los países en desarrollo. Aunque el mismo se concibió originalmente como una instancia para asesorar la formación de cuadros nacionales para la defensa legal internacional del Estado, hoy se ha convertido en un consultor legal que puede ofrecer a precios más accesibles servicios legales especializados, teniendo como único inconveniente que para un caso concreto solo puede ofrecer sus servicios respecto de uno de los Estados parte (PHAM, 2004).

Así mismo, existe un límite económico relacionado con la presentación de un caso en la sede 
internacional de los organismos. Mas se mencionó con anterioridad que el procedimiento facilita el acceso, la aceptación real de un caso depende del acompañamiento y presencia de un abogado que represente al Estado. Un tercer nivel de obstáculos volviendo al ámbito estatal tiene que ver con la ausencia de espacios efectivos de coordinación para la acción o respuesta legal en los ámbitos globales analizados.

El tema de la asistencia legal, aparte de su dimensión económica ligada con sus costos, tiene una especificidad en cuanto a su disponibilidad. Si bien algunos países del tercer mundo cuentan con una importante experiencia de abogados(as) en el acceso y manejo del Sistema Interamericano, no ocurre lo mismo en el campo del derecho económico. Como se sostiene en los distintos estudios, a nivel académico existe una formación más o menos profunda sobre los organismos aquí analizados (Gómez, 2009a \& Francke, 2007). Sin embargo, las firmas de abogados(as) afincadas en este país no cuentan con experiencia concreta en llevar casos al CIADI o al organismo de solución de controversias de la OMC. Lo anterior, aunado a la ausencia de un verdadero sistema de defensa legal del Estado, explica porque se termina apelando a firmas internacionales de abogados, lo cual encarece los costos de ingreso y uso de determinado sistema de solución de conflictos.

En último lugar y en relación con esta parte previa al inicio formal de una causa, otro obstáculo para el acceso remite al idioma reglamentario $\mathrm{y} / \mathrm{o}$ fáctico en que las causas deben presentarse $y$ tramitarse. Para el CIADI parece no haber mayor inconveniente si se tiene en cuenta que el español es uno de Ios idiomas oficiales en que un caso puede expresarse. Caso diferente se presenta con el OSD, el cual si bien oficialmente permite el uso de varias lenguas, en la práctica muchos de los casos se deben tramitar en inglés y eventualmente en francés.

\section{Acceso a la justicia durante el desarrollo de un caso}

Luego de la admisión del caso, el acceso a la justicia se evaluó teniendo en cuenta variables identificadas como particulares del trámite de un proceso en cuanto tal. Sobre estas últimas se valoraron, entre otras, la agilidad del proceso, el respeto de los derechos, el acceso a recursos, etc.

En materia de oportunidades que se pueden visualizar como transversales, los procedimientos que guían o enmarcan el desarrollo de una causa son no sólo flexibles, sino ágiles en cuanto establecen períodos claros para su desarrollo. Obviamente, como se verá en otros acápites de este documento, el cumplimiento está afectado en su celeridad por distintos aspectos procesales que afectan el mismo. A este nivel es importante resaltar que para el caso de la OMC, si bien el procedimiento es claro y en principio cumple los tiempos, su desarrollo es confidencial (Gómez, 2009a).

Como parte de este plano procedimental, algunos de ellos permiten el traslado de las audiencias o del proceso entero a zonas diferentes a su sede. Esto ocurre en particular con el CIADI y con el arbitraje comercial internacional, aunque se debe tener en cuenta que para el primer caso, quien puede escoger el foro es la empresa privada y no el Estado. Sin embargo, la rigidez espacial se mantiene para los casos de la OMC.

Un tercer aspecto, éste sin más general y que se visualizó como oportunidad, tuvo que ver con el tratamiento técnico del conflicto. En sentido amplio, todas las instancias cuentan con personas especialistas en los temas objeto de la controversia. Esto es resaltable si tenemos en cuenta que muchos de estos conflictos presentan una especificidad y complejidad técnica que es importante si se le compara con las atribuciones más amplias de un juez de la judicatura nacional. 
Ahora bien, los obstáculos centrales identificables durante el trámite se pueden agrupar en tres planos. De un lado están los costos, los cuales a su vez se desglosan en tres planos. En primer lugar el costo de acopiar y presentar las pruebas. Como se anotó en el anterior punto, el tipo de conflictos ventilados en las instancias aquí estudiadas conllevan temas sumamente técnicos, sociales y económicamente sensibles. Por tanto, para el caso de los Estados y sus abogados, el costo de recoger y presentar pruebas conlleva la asesoría y el apoyo de expertos en determinados temas, los cuales en muchos casos forman parte de los servicios de las grandes firmas de abogados. Esto es particularmente evidente en los ámbitos de justicia global económica (Shaffer, 1993).

Como se había indicado en el anterior acápite, el idioma es en algunos casos un limitante para ingresar al sistema de solución de controversias. Sin embargo, este puede ser enfrentado a lo largo del desarrollo de un proceso mediante alguno de los sistemas de traducción altamente técnicos con que cuentan algunas de las instancias estudiadas. El punto, en materia de acceso, es que éste cuesta en cuanto el mecanismo de traducciones, por lo general, no está subvencionado y es pagado a precios de mercado.

En definitiva y como se enunció en el numeral anterior, el costo de la asesoría legal es igualmente un potencial obstáculo. Si bien el costo real de su asesoría y acompañamiento técnico no siempre es público, los datos recogidos en las investigaciones muestran que su costo es por lo general elevado. A este respecto, surge siempre la inquietud de si unos determinados costos, generalmente, internacionales de asistencia legal, son altos o excesivos para un Estado de desarrollo medio si tenemos en cuenta los costos que asume para el pago de determinadas asesorías o asistencia legal a nivel interno (Duclos, 2009).
Un segundo plano de obstáculos tiene que ver con la calidad de los árbitros o decisores finales en torno de un conflicto. En general la investigación mostró que en el plano del derecho internacional económico, el sistema de designación de árbitros funciona bajo un sistema denominado coloquialmente "carrusel". Esto significa que dada la estructura cerrada y elitista de las listas de árbitros especialistas en distintos temas y el hecho de que muchos de ellos son a su vez miembros de firmas de abogados; en la práctica, las personas que sirven de árbitros en un momento determinado, en el pasado han sido, o podrán ser en el futuro, asesores de las empresas o Estados que están inmersos en un conflicto. Lo anterior impacta en lo que comúnmente se han identificado como dinámicas sesgadas de los árbitros. En efecto, cuando un decisor ha sido a su vez en el pasado un asesor de un Estado o una empresa, surgen inquietudes sobre su capacidad de imparcialidad y de hecho se ha demostrado que en algunos casos no ha existido, como se mencionó específicamente con referencia al CIADI (Horn, et ál., 1999).

Otro aspecto tiene que ver con el sesgo de "origen" de los decisores. Si bien en algunas instancias como la OMC se prevé el nombramiento de un miembro del panel procedente de países en desarrollo $y$, en general, se pide tener en cuenta las condiciones de estos países, cuando estos están involucrados en un conflicto, en el caso del CIADI y el arbitraje comercial esto no es necesario. De esta forma, en la práctica los árbitros tienden a provenir del mundo desarrollado, lo cual en algunos casos les ha dificultado entender y valorar determinadas medidas tomadas por los Estados que pueden considerarse no solo legales sino legítimas en contextos del mundo en desarrollo (Frank, 2009).

En conclusión, como obstáculos se identificó el tema de los recursos. En líneas generales la justicia internacional no admite fácilmente la figura de los 
recursos de alzada. Lo anterior se ha considerado históricamente una ventaja de estas instancias. En efecto, la ausencia de formulas de apelación se ha justificado por el carácter excepcional de estos espacios, por su agilidad y por la índole profundamente técnica de sus fallos. Algunos han sostenido que introducir una estructura de doble instancia en ámbitos como los arbitrales, por ejemplo, es como eliminar una de sus ventajas comparativas más claras. Con todo, visto en términos de acceso la posibilidad de una doble instancia es una garantía que debe permitir no sólo decisiones a tiempo, sino correctas a la luz del derecho aplicable (Hugges, 2003 \& Tsatsos, 2009). Para nuestros objetos de estudio, las decisiones del OSD de la OMC admiten apelación mientras las del CIADI son anulables.

\section{Acceso a la justicia con posterioridad a la decisión de un caso}

El marco analítico desarrollado en esta investigación, incluyó el momento posterior a una decisión de solución de un conflicto, en cuanto si ésta no se obtiene, no se cumple o es incompleta, afecta las expectativas de justiciabilidad de las partes inmersas en un conflicto.

En este plano las oportunidades identificadas fueron tres. En primer lugar, las instancias analizadas toman decisiones cercanas a los tiempos o apegadas a los períodos establecidos en sus procedimientos. Por tanto, la justicia obtenida es en cierto sentido pronta. Adicionalmente, los organismos analizados toman en general decisiones de fondo. Es decir, no es común que se inhiban en el tratamiento de un caso por razones estrictamente formales. En definitiva, las decisiones tienen un carácter obligatorio basado en el principio de la buena fe reconocido en la carta de la ONU. En este último sentido y como se mencionó en la parte uno de este artículo, las decisiones de la OMC pueden hacerse obligatorias mediante el régimen de sanciones económicas previsto
(Horlick \& Mizulin 2005); las decisiones del CIADI, pueden reconocerse como decisiones judiciales internas sin necesidad del exequátur, mientras las de arbitraje internacional si precisan de este último para su incorporación interna en caso de no cumplimiento.

Los obstáculos en la etapa última a partir de la cual analizamos el acceso son dos en lo fundamental. De un lado, el procedimiento posterior que puede ligarse al cumplimiento final de una decisión global de resolución de conflictos, no facilita el acatamiento de una decisión en el tiempo esperado. Como muestran varios casos emblemáticos, como el del banano, por ejemplo, las decisiones del OSD de la OMC no se han cumplido o se han prolongado en el tiempo, ocurriendo lo mismo respecto del CIADI cuyas decisiones efectivas quedan inmersas en algunos casos en procesos de negociación entre el Estado y la empresa privada aunque en líneas generales aquellos casos que llegan a una decisión final tienden a cumplirse (Jackson, 2000 \& Fonseca, 2004).

Un aspecto particular de esto último y como segundo factor de dificultad tiene que ver con la judicialización nacional de decisiones tomadas foráneamente. Este sería el caso de los laudos de arbitraje comercial con participación del Estado, los cuales en buena parte de los casos, donde ha participado Colombia, han terminado siendo anulados por decisiones judiciales de cortes nacionales. Esto si bien puede tener justificaciones legales profundamente válidas y expresar intereses nacionales, visto desde el acceso puede ser un factor crítico para permitir la efectividad del mismo.

\section{CONCLUSIONES}

La justicia internacional está creciendo, sea en la cantidad de instancias resolutorias de conflictos; sea en el tipo de sujetos cuyas causas son admisibles, el cual incluye hoy en determinados niveles a los individuos; sea en la capacidad de llegar 
a decisiones "vinculantes" para las partes. En el contexto de la globalización, el fortalecimiento de la justicia internacional parece claro y en determinados ámbitos deseable.

El fortalecimiento de la justicia internacional, no ha cuestionado del todo su carácter excepcional, centrado en buena parte en los Estados y accesible a muy poco tipo de sujetos. Quizás por lo anterior, la preocupación por el acceso a la justicia internacional es limitada, aunque como se expresa en este artículo es creciente y cada vez más contundente.

El acceso a la justicia, sea a nivel nacional o internacional debe entenderse como la posibilidad no sólo de llevar un caso a un trámite institucional, sino de contar con una decisión con plenas garantías y que permita resolver de fondo un conflicto, lo cual tiene que ver con la efectividad de una decisión. En este sentido, el acceso a la justicia debe verse de manera integral. El presente artículo ofrece un marco analítico y un conjunto de variables que pretenden dar cuenta de la complejidad de este acceso.

A partir de lo anterior, se identificaron un conjunto de hipótesis gruesas sobre las oportunidades y obstáculos para el acceso de los países del mundo en desarrollo a instancias como el CIADI del Banco Mundial y el OSD de la OMC. En tal sentido, se plantearon, entre otros, que las principales oportunidades están en la flexibilidad de los procesos de admisión y desarrollo de un caso; en el nivel de expertise técnico que sustenta una decisión, en la rapidez relativa del desarrollo de un caso $y$ en el nivel de eficacia igualmente relativo de decisiones de fondo.

Los obstáculos por su parte se ubicaron en el terreno de los costos de asistencia legal y de acopio de pruebas; en la ausencia de una formación idónea al interior de los Estados del tercer mundo para responder o liderar casos en las instancias estudiadas, así como en la respectiva falta de coordinación intraestatal. Así mismo, se identificaron problemas relacionados con la potencial falta de imparcialidad e independencia por parte de los decisores en ambas instancias, así como la ausencia parcial de recursos de alzada contra las decisiones.

El presente trabajo es un primer avance de un proceso de investigación que profundizará en las anteriores hipótesis, particularmente, mediante su mayor concreción y su validación empírica, en especial a partir del análisis de la situación de algunos países, incluyendo el caso colombiano.

\section{REFERENCIAS}

Acosta, P. (2007). El derecho de acceso a la justicia en la jurisprudencia interamericana. Bogotá: Universidad Externado de Colombia.

Arenas, M. (s.f.). Los obstáculos de los países en desarrollo en el Sistema de Solución de Controversias de la OMC. Tesis de Maestría en Derecho. Bogotá: Universidad de los Andes.

Bou, M. (2004). El sistema de justicia en América Latina, entre las reformas y la inercia institucional. Barcelona: Instituto Internacional de Gobernabilidad.

Capelleti \& Garth. (1996). El acceso a la justicia. México: Fondo de Cultura Económico.

CIDH. (2007). El Acceso a la justicia como garantía de los derechos económicos, sociales y culturales. Documento oficial, No 4.

Cruz, L. (2002, enero). Las comunicaciones Amicus Curiae en el mecanismo de solución de diferencias de la Organización Mundial del Comercio: el asunto Amianto. En: Revista Española de Derecho Internacional. LIV (1).

DPLF (2007). Barreras para el acceso a la justicia en América Latina. Documento presentado ante la Comisión Interamericana de los Derechos Humanos. Washington. 
Domingo, P. et ál. (2001). Rule of Law in Latin America. the International Promotion of Judicial Reform. London: Institute of Latin American Studies.

Duclos, N. (2009, septiembre). El contrato de Estado y el arbitraje de inversión. El debate sobre la Clausula Paraguas. Conferencia dictada en el IV Seminario de Arbitraje Internacional de Inversión. Universidad Externado de Colombia. Bogotá.

Frank, S. (2009). Development and Outcomes of Investment Treaty Arbitaration. In: Harvard International Law Journal. 50 Harv. Int'l J. 435.

Fonseca, A. (2004). La guerra comercial del plátano. Una aproximación desde la teoría de juegos. En: Estudios Agrosociales y Pesqueros. 201.

Francke, M. (2007). Chile's Participation in the Dispute Settlement System: Impact on Capacity Building. Documento borrador del proyecto WTO Dispute Settlement and Sustainable Development.

Gómez, G. (2009a). La efectividad de las decisiones del OSD. Madrid. Mimeo.

Gómez, G. (2009b). Decisiones del OSD, Derechos Humanos y desarrollo. Madrid: Mimeo.

Horlick, G. \& Mizulin, N. (2005). Los países en desarrollo y el mecanismo de solución de controversias de la OMC. En: Integración y Comercio. 23.

Hernández, O. (2009). Oportunidades y obstáculos para el acceso del Estado al arbitraje de inversión. Documento interno de investigación. Diciembre, 2009.

Horn, H.; Mavroidis, P. \& Nordstrom, H. (1999, diciembre). Is the Use of the WTO Dispute Settlement System Biased? In: International Trade. Center for Economic Policy Research. Discussion Paper, No. 2340.
Hughes, V. (2003). El sistema de solución de diferencias de la $\mathrm{OMC}$, una experiencia exitosa.

Jackson, J. (2000). The Role and Effectiveness of the WTO Dispute Settlement Mechanism. In. Brooking Trade Forum.

Moncayo, H. (1995). Acceso de los Pobres a la Justicia en Colombia. En: Franz Vanderschueren and Enrique Oviedo (Eds.) Acceso de los Pobres a la Justicia, Ediciones Sur/PNUD. Santiago de Chile.

Moreno, L. (2000). Acceso a la justicia. Bogotá: Academia Colombiana de Jurisprudencia.

Odumosu, T. (2007). The Law and Politics of Engaging Resistance in Investment Dispute Settlement. The Dickinson School of Law of the Pennsylvania State University, Carlisle, PA. Penn State International Law Review. Fall, 26 Penn St. Int'I L. Rev. 251.

Peterson, E. (2003). Emerging Bilateral Investment Treaty Arbitration and Sustainable Development (Research note). International Institute for Sustainable Development IISD. recovered from [http:// www.iisd.org/pdf/2003/trade_bits_disputes.pdf].

Pham, H. (s.f.). Developing Countries and the WTO: The Need for More Mediation in the DSU. Online document. In: Harvard Negotiation Law Review. 9.

Shafer, G (2003). How to make the Wto Dispute Settlement Work for developing countries. Some Proactive strategies. ICTSD Resource Paper, No. 5.

Smith, R (2007). Human Rights and Access to Justice. In: International Journal of the Legal profession, Vol. 14, No. 3.

Tsatsos, A. (2009). ICSID Jurisprudence: Between Homogeneity and Heterogeneity. A Call for Appeal? Transnational Dispute Management (TDM) 6 (1). 
UNDP (2004). Access to Justices. Practice Note. Online document .
Vigoriti, V. (2008). Acceso a la justicia, sistemas alternativos de solución de conflictos. Perspectivas. En: Anuario de Justicia Alternativa, 9. 